\title{
T. Tasso, Osvoboždennyj Ierusalim: poèma, perevod s ital. i primeč. R. Dubrovkina, Izdatel'stvo Ivana Limbacha, Sankt-Peterburg 2020, pp. 607.
}

La nuova traduzione russa della Gerusalemme Liberata di Tasso, condotta da Roman Dubrovkin e pubblicata nel 2020 per l'editore Ivan Limbach, viene a colmare un vuoto nella ricezione della poesia italiana in Russia. Lo osserva Dubrovkin nella postfazione che chiude il volume ("Tam, gde končaetsja slovar....”. Ot perevodčika), in cui ripercorre per sommi capi la storia della fortuna (e della sfortuna) russa del poema tassiano: a partire dalla grande notorietà raggiunta nel primo Ottocento, che viene meno nella seconda metà del secolo; passando per tre versioni edite a inizio Novecento, tradotte da D.E. Min, O. Golovin (pseudonimo di R.F. Brandt) e V.S. Lichačev; per arrivare infine all'oblio dell'epoca sovietica, durante la quale per motivi ideologici (anti-religiosi e politici) una traduzione integrale sarebbe stata impensabile e alcuni traduttori poterono pubblicarne alcuni frammenti solo celandoli all' interno di corpose antologie. Solo nel 2007 , infine, l'editore accademico Nauka ha ripubblicato la versione in pentametri giambici sciolti di V.S. Lichačev, corredata da un ampio apparato di commento (pp. 620-713) a cura di A.O. Demin. Dubrovkin individua alcuni punti deboli in tutte le traduzioni esistenti - ora perché troppo remote, ora per motivi linguistici, formali o estetici - e propone per la prima volta dopo più di un secolo una nuova traduzione integrale del poema.

Il volume è aperto da un'introduzione, anch'essa a firma di Dubrovkin, nella quale assieme alle principali coordinate critiche che inquadrano la Liberata e ad alcuni dati sulla sua fortuna in Europa, il lettore russo viene a conoscenza della complessa storia della stesura e delle edizioni del poema: poiché di fatto non esiste una versione 'definitiva' autorizzata da Tasso per la stampa, la Liberata pone spinose questioni filologiche su cui i ricercatori lavorano da lunghi anni. In una nota a chiusura del volume si apprende che la traduzione, a differenza delle versioni precedenti (dell'Ottocento e del primo Novecento), ha potuto basarsi sulle edizioni più accurate del testo, frutto delle ricerche filologiche più recenti: in particolare su quella curata da Lanfranco Caretti, ad oggi ritenuta la più attendibile anche dagli studiosi italiani. Il traduttore, tuttavia, dichiara di aver ritenuto "inopportuno attenersi rigidamente alla lettera di una qualche concreta edizione, pur affidandosi nella schiacciante maggioranza dei casi alla redazione di Caretti” ( . 554) - ciò che solleva alcuni problemi sotto il profilo della correttezza filologica: non vengono esplicitati i luoghi del testo in cui il traduttore si è discostato dall'edizione di Caretti, né quali altre edizioni sono state consultate per questi passi.

Chiudono infine il volume un indice dei nomi e un breve riassunto di ogni canto che aiutano il lettore a orientarsi nel poema; e un apparato di commento, per la verità piuttosto contenuto (pp. 
555-574) rispetto all'edizione del 2007 di Nauka o alle edizioni italiane del poema, che consta principalmente di note esplicative di natura storica o geografica.

Quanto alla traduzione vera e propria, che nel volume è priva del testo a fronte, Dubrovkin si muove nella direzione opposta rispetto alla rottura della metrica sillabo-tonica sperimentata negli ultimi decenni in alcune traduzioni di poesia italiana in russo (traduzioni in prosa, in versi liberi o in versi sillabici da Ariosto o Dante, ad esempio). Il traduttore riproduce la forma del poema originale, facendo corrispondere alle ottave in endecasillabi della Liberata ottave russe in pentametri giambici, secondo lo schema rimico авававсc (le posizioni delle rime maschili e femminili si invertono a ogni ottava). Il ritmo del pentametro giambico - che in Russia è stato spesso usato come equivalente dell'endecasillabo italiano (un esempio su tutti, la celebre traduzione di M.L. Lozinskij della Commedia dantesca) - è molto vicino alla prosodia degli endecasillabi della Liberata, i quali, lo rilevano gli studi più recenti, sono vincolati a poche tipologie ritmiche, in cui quella giambica è una delle più frequenti. Il punto di maggior distanza tra la forma italiana e quella russa, come osserva lo stesso traduttore, risiede piuttosto nella tradizione legata all'ottava: giacché quella russa si è sviluppata principalmente come forma lirica, Dubrovkin ha dovuto cercare un modo per ricondurla al modello epico plasmato da Tasso.

Preservando nella maggioranza dei casi l'autoconclusività delle ottave della Liberata, Dubrovkin ha potuto conservare il particolare ritmo sincopato della narrazione. Anche la struttura interna dell'ottava tassiana segmentata in misure pari - l'unità di base sono i distici, a loro volta aggregati in due quartine: $4(2+2)+4(2+2)$ - è rispettata, come si evince osservando anche solo i segni di interpunzione a fine verso: particolare attenzione è rivolta alla conservazione della pausa forte tra quarto e quinto verso (sebbene non manchino le eccezioni, si veda come unico esempio il forte enjambement in XII, 4I).

Pur non potendo in questa sede condurre un'analisi puntuale, si può osservare come anche l'andamento della sintassi - libera di disporsi a suo piacimento all' interno della struttura simmetrica dell'ottava tassiana - venga in gran parte preservato: nella traduzione, come del resto nell'originale, sono frequenti gli enjambement e i sommovimenti nell'ordine delle parole (iperbati, epifrasi, anastrofi etc.), anche se, per via dell'asimmetria sintattica tra la lingua russa e quella italiana, l'effetto prodotto è forse meno potente rispetto al testo originale. Cadono invece talvolta le figure di ripetizione, frequenti nella Liberata, ciò che comporta la perdita dell'effetto patetico ricercato da Tasso: è ad esempio notevole la differenza di tono quando Dubrovkin riesce a conservare l'anadiplosi (XII, 52-53) e quando invece cade (XVI, 60-6I).

Rimane in ogni caso costante lo sforzo di Dubrovkin per conservare lo stilema più caratteristico della Liberata, che in una lettera dell'ottobre 1575 Tasso stesso definiva "parlar disgiunto": ossia, sintetizzando e semplificando molto, l'andamento paratattico e asindetico che caratterizza il poema. Sul fronte della punteggiatura, invece, osserviamo nella traduzione un'abbondanza di punti esclamativi (in numero molto superiore rispetto alle edizioni italiane del poema, cfr. XX, I32-I34), forse riconducibili all'esigenza di ricostituire la dizione patetica perduta con la riduzione delle figure di ripetizione.

La parte più complicata della traduzione - portata a termine in I4 anni di lavoro dal 2006 al 2020 - è piuttosto quella legata al lessico: se infatti è (relativamente) possibile restituire la forma metrico-rimica e la sintassi dell'originale, è molto più complesso riprodurre le stratificazioni semantiche del lessico cinquecentesco di Tasso. D'altronde la tradizione poetica italiana e quella russa si sono sviluppate con alcune significative differenze: ad esempio anche attraverso Tasso la lingua della poesia italiana ha potuto accogliere elementi di cruda violenza e brutalità nelle descrizioni delle 
battaglie, i quali, lo nota lo stesso Dubrovkin, sono quasi assenti nella tradizione russa. Per fornirsi di un modello approssimativo in questo senso, il traduttore dichiara di aver tenuto presente tra gli altri anche la novecentesca Usobica di Maksimilian Vološin.

Di questa discrepanza lessicale si può giudicare osservando cosa accade, nella traduzione, a un lemma fondamentale come "errare", che con la sua polisemia (vagare, pellegrinare, peccare etc.) rappresenta una categoria fondamentale del poema, molto complessa da rendere in russo. Nessuno dei termini utilizzati da Dubrovkin (brodit', skitat'sja, palomnik, piligrim, kočevoj etc.) può restituire la polisemia dei termini originali, tanto che talvolta il traduttore li omette, li sostituisce con perifrasi o li traduce con espressioni anche lontane da quelle di partenza. E tuttavia, sul piano generale, il traduttore riesce a mantenere costantemente lo stile solenne e "magniloquente" dell'originale.

Del resto la concezione traduttiva di Dubrovkin non è orientata né verso una letteralità $[d o-$ slovnost'] strettamente intesa, né verso i parametri di un'edizione accademica (lo dimostra già l'esiguità del commento), bensì verso una resa esteticamente valida della poesia originale e una sua riespressione attraverso gli strumenti di una diversa lingua poetica. Dunque, nonostante le inevitabili perdite sul piano della precisione lessicale, sono numerosi i passi di sicura riuscita della traduzione, specie i più celebri del poema tassiano, come il combattimento tra Tancredi e Clorinda (XII, 52-70), o ancora i notturni (II, 96-97; VI, I03-IO5). Di uno dei notturni più noti riportiamo infine un'ottava, in qualità di esempio dei risultati raggiunti da Dubrovkin:

Era la notte, e 'l suo stellato velo chiaro spiegava e senza nube alcuna, e già spargea rai luminosi e gelo di vive perle la sorgente luna. L'innamorata donna iva co 'l cielo le sue fiamme sfogando ad una ad una, e secretari del suo amore antico fea i muti campi e quel silenzio amico.
Ауна возникца из-за перевала, Окаймкена безоблачною тьмой, Роняя жемчуга на покрывало, Жемчужною играя бахромой. Свой жар сердечный дева отдавала Ночным полям и темноте немой Свидетелям мюбви своей нетленной Аетела к свету вместе со вселенной. 